\title{
Supplementary Material \\ for \\ Contributions of Nordic anthropogenic emissions on air pollution and premature mortality over the Nordic region and the Arctic
}

Ulas $\operatorname{Im}^{1,2}$, Jesper H. Christensen ${ }^{1,2}$, Ole-Kenneth Nielsen ${ }^{1,2}$, Maria Sand ${ }^{3}$, Risto Makkonen ${ }^{4,5}$, Camilla Geels $^{1,2}$, Camilla Anderson ${ }^{6}$, Jaakko Kukkonen ${ }^{4}$, Susana Lopez-Aparicio ${ }^{7}$, Jørgen Brandt ${ }^{1,2}$

1 Aarhus University, Department of Environmental Science, Atmospheric Modelling Section, Frederiksborgvej 399, Roskilde, Denmark.

2 Interdisciplinary Center for Climate Change (iCLIMATE), Frederiksborgvej 399, Roskilde, Denmark.

3 Center for International Climate Research, Postboks 1129 Blindern, 0318 Oslo, Norway. 4 Finnish Meteorological Institute, Erik Palmenin aukio 1, P.O.Box 503, FI-00101, Helsinki, Finland.

5 University of Helsinki, Institute for Atmospheric and Earth System Research, P.O. Box 64, 00014, Helsinki, Finland.

6 Swedish Meteorological and Hydrological Institute, SE-60176 Norrköping, Sweden.

7 NILU - Norwegian Institute for Air Research, Instituttveien 18, P.O. Box 100, 2027 Kjeller, Norway.

Table S1. Geographical information of the measurement stations used for model evaluation.

\begin{tabular}{|l|c|c|l|}
\hline Station Name & Longitude & Latitude & Station Type \\
\hline \multicolumn{3}{|c|}{ Denmark } \\
\hline Anholt & 11.52 & 56.72 & Regional background \\
\hline Ulborg & 8.43 & 56.29 & Regional background \\
\hline Keldsnor & 10.74 & 54.75 & Regional background \\
\hline Risø & 12.09 & 55.69 & Regional background \\
\hline Odense Rådhus & 10.39 & 55.40 & Urban background \\
\hline Aarhus Botanical Garden & 10.19 & 56.16 & Urban background \\
\hline Aalborg Østerbro & 9.93 & 57.05 & Urban background \\
\hline Copenhagen HCØ & 12.56 & 55.70 & Urban background \\
\hline & & & \\
\hline Luukki & 24.68 & 60.31 & Regional background \\
\hline Oulanka & 29.40 & 66.32 & Regional background \\
\hline Sammaltunturi & 24.11 & 67.97 & Regional background \\
\hline Uto & 21.37 & 59.78 & Regional background \\
\hline Virolahti & 27.67 & 60.53 & Regional background \\
\hline Virolahti_2 & 27.68 & 60.53 & Regional background \\
\hline Ahtari_2 & 24.19 & 62.59 & Regional background \\
\hline Kallio_2 & 24.95 & 60.19 & Urban background \\
\hline Kasarmipuisto & 27.67 & 62.89 & Urban background \\
\hline
\end{tabular}




\begin{tabular}{|c|c|c|c|}
\hline Kisapuisto & 25.65 & 60.99 & Urban background \\
\hline \multicolumn{4}{|c|}{ Norway } \\
\hline Birkenesobservatoriet & 8.25 & 58.39 & Regional background \\
\hline Haukenes & 9.49 & 59.20 & Regional background \\
\hline Hurdal25 & 11.07 & 60.37 & Regional background \\
\hline Kårvatn & 8.88 & 62.78 & Regional background \\
\hline Lommedalen & 10.49 & 59.95 & Regional background \\
\hline Prestebakke & 11.53 & 59.00 & Regional background \\
\hline Sandve & 5.20 & 59.20 & Regional background \\
\hline Tustervatn & 13.91 & 65.83 & Regional background \\
\hline Grønland & 10.76 & 59.91 & Urban background \\
\hline Rådhuset & 5.33 & 60.39 & Urban background \\
\hline \multicolumn{4}{|c|}{ Sweden } \\
\hline Aspvreten & 17.38 & 58.80 & Regional background \\
\hline Bredkalen & 15.34 & 63.85 & Regional background \\
\hline Esrange & 21.07 & 67.88 & Regional background \\
\hline Hoburgen & 18.15 & 56.92 & Regional background \\
\hline Rorvik & 11.94 & 57.41 & Regional background \\
\hline Raao & 11.91 & 57.39 & Regional background \\
\hline Vavihill & 13.15 & 56.03 & Regional background \\
\hline Grimso & 15.47 & 59.73 & Regional background \\
\hline Norra_Kvill & 15.56 & 57.81 & Regional background \\
\hline Norr_Malma & 18.63 & 59.83 & Regional background \\
\hline Ostad & 12.40 & 57.95 & Regional background \\
\hline Vavihill & 13.15 & 56.03 & Regional background \\
\hline Vindeln & 19.77 & 64.25 & Regional background \\
\hline Goteborg_Femman & 11.97 & 57.71 & Urban background \\
\hline Malmo_Raadhuset & 13.00 & 55.61 & Urban background \\
\hline Norrkoping_Rosen & 16.19 & 58.59 & Urban background \\
\hline Stockholm_Torkel_Knutssongatan & 18.06 & 59.32 & Urban background \\
\hline Umeaa_Stadsbiblioteket & 20.27 & 63.83 & Urban background \\
\hline Vasteraas_Stadshuset & 16.55 & 59.61 & Urban background \\
\hline
\end{tabular}


Table S2. Exposure-response functions (ERF) used in EVA to calculate premature mortality.

\begin{tabular}{|l|l|l|}
\hline \multirow{2}{*}{ Health effects (compounds) } & Exposure-response coefficient & \multirow{2}{*}{ Valuation, $\boldsymbol{\epsilon}_{\mathbf{2 0 1 3}}$} \\
\cline { 2 - 3 } & $(\boldsymbol{\alpha})$ & \multirow{2}{*}{ (EU27) } \\
\hline Acute mortality ${ }^{2,3}\left(\mathrm{SO}_{2}\right)$ & $7.85 \mathrm{E}-6 \mathrm{cases} / \mu \mathrm{gm}^{-3}$ & \multirow{2}{*}{532,099 per case } \\
\hline Acute mortality ${ }^{2,3}\left(\mathrm{O}_{3}\right)$ & $3.27 \mathrm{E}-6^{*} \mathrm{SOMO} 35 \mathrm{cases} / \mu \mathrm{gm}^{-3}$ & \\
\hline Chronic mortality $1,4, \mathrm{YOLL}(\mathrm{PM})$ & $1.138 \mathrm{E}-3 \mathrm{YOLL} / \mu \mathrm{gm}^{-3}(>30$ years $)$ & 57,510 per YOLL \\
\hline Infant mortality ${ }^{5}, \mathrm{IM}(\mathrm{PM})$ & $6.68 \mathrm{E}-6 \mathrm{cases} / \mu \mathrm{gmm}^{-3}(>9$ months $)$ & $2,298,148$ per case \\
\hline
\end{tabular}

${ }^{1}$ Pope et al. (2002), ${ }^{2}$ Anderson (1996), ${ }^{3}$ Touloumi (1996), ${ }^{4}$ Pope et al. (1995), ${ }^{5}$ Woodruff et al. (1997).
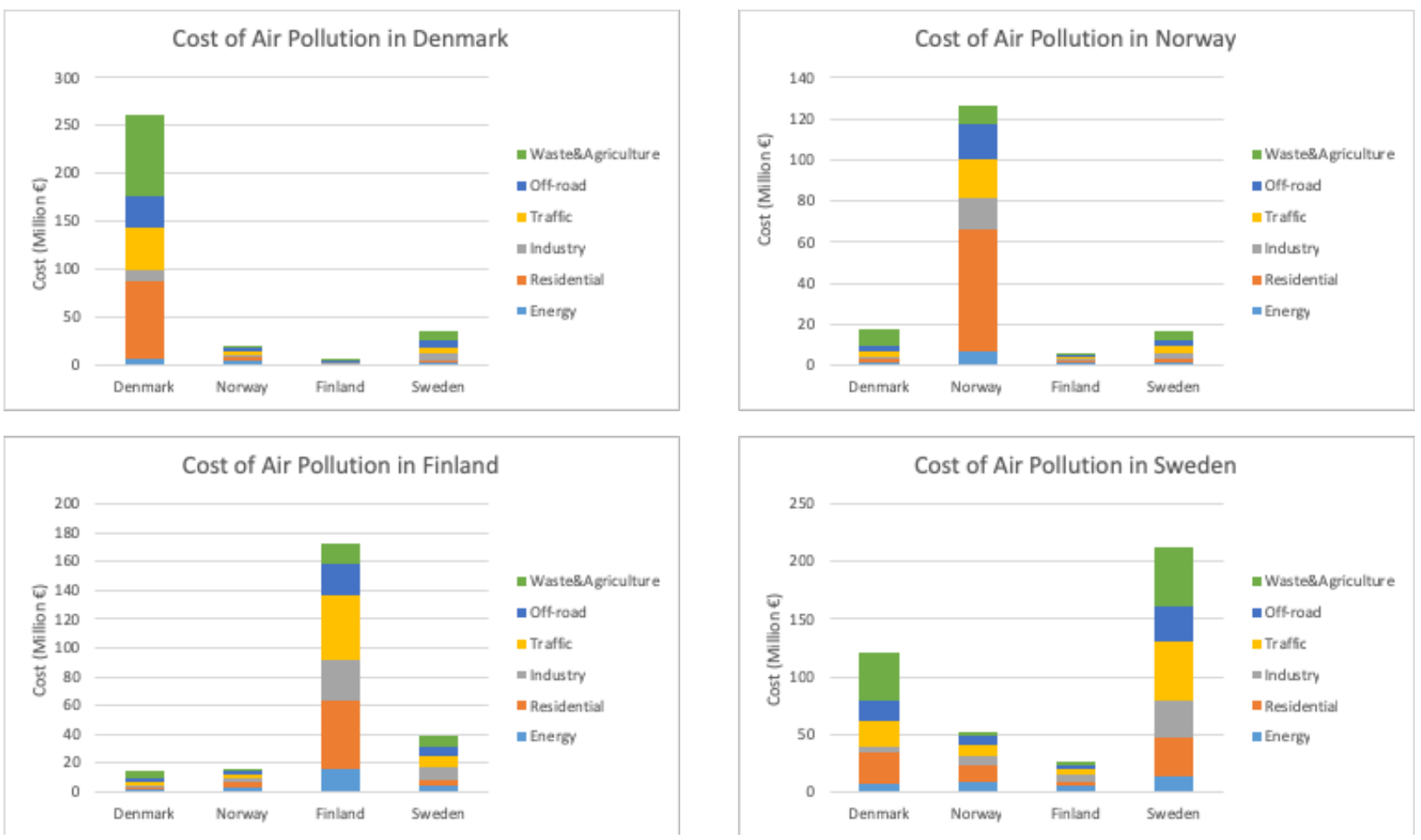

Fig. S1. Sectoral contribution of emissions to health-related costs in a) Denmark, b) Norway, c) Finland, and d) Sweden.

\section{REFERENCES}

Anderson, H.R., Ponce de Leon, A., Bland, J.M., Bower, J.S. and Strachan, D.P. Air Pollution and daily mortality in London: 1987-92. British Medical Journal, 312, 665-669, 1996. 
Pope, C.A., Burnett, R.T., Thun, M.J., Calle, E.E., Krewski, D., Ito, K. and Thurston, G.D. Lung cancer, cardiopulmonary mortality and long-term exposure to fine particulate air pollution. Journal of American Medical Association, 287 (9), 1132-1141, 2002.

Pope, C.A. Thun, M.J., Namboodiri, M.M., Dockery, D.W., Evans, J.S., Speizer, F.E. and Heath Jr, C.W. Particulate air pollution as a predictor of mortality in a prospective study of US adults. American Journal of Respiratory and Critical Care Medicine, 151, 669-674, 1995.

Touloumi, G., Samoli, E. and Katsuyanni, K. Daily mortality and "winter type" air pollution in Athens, Greece - a time series analysis within the APHEA project. Journal of Epidemiology and Community Health, 50 (suppl 1), S47 - S51, 1996.

Woodruff, T.J., Grillo, J. and Schoendorf, K.C. The relationship between selected causes of postneonatal infant mortality and particulate air pollution in the United States. Environmental Health Perspectives, 105, 608-612, 1997. 